\title{
The mutual alteration of bacterial susceptibility to antimicrobials in dual- species biofilm
}

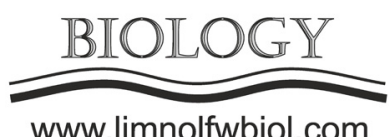

\author{
Trizna E.Y., Baidamshina D.R., Mironova A.V., Kayumov A.R.* \\ Kazan Federal University, Kremlevskaya Str., 18, Kazan, 420008, Russia
}

\begin{abstract}
The biofilm formation by pathogenic bacteria significantly reduces the efficacy of antimicrobial therapy, leads to chronic diseases and slows down the wounds healing. Many infectious processes are caused rather by consortium of bacteria than by one microorganism. As a result, mixed bacterial biofilms are formed, in which the resistance to antimicrobials increases due to the survival of some microorganisms in the biofilm of resistant strains and the transition to an uncultivated state. Here we show that the bacterial susceptibility to antimicrobials in dual-species biofilms significantly differs from monoculture biofilms depending on both conditions and chosen antimicrobial agents. While Staphylococcus aureus could completely avoid some antimicrobials by embedding into the biofilm of various gram-negative bacteria, the very same consortium was characterized by 10 -fold increase in susceptibility to broad-spectrum antimicrobials like ciprofloxacin and aminoglycosides compared to monocultures. These data clearly indicate that efficient treatment of biofilm-associated mixed infections requires antimicrobials active against both pathogens, since the interbacterial antagonism would enhance the efficacy of treatment.
\end{abstract}

Keywords: Microbial biofilms, susceptibility to antimicrobials, interbacterial interactions, bacteria.

\section{Introduction}

While in the biofilm, bacteria are extremely resistant to bactericidal drugs, antibiotics and the immune system of the host, and survive at antibiotics concentrations of 500-1000 times greater than their minimum inhibitory concentration in vitro (SanchezVizuete et al., 2015). Despite of active investigation of monomicrobial biofilms, studies of mixed communities have been made only for several bacteria, for example, $S$. aureus $-P$. aeruginosa biofilms for which the mutual changes in antibiotic resistance driven by various metabolies synthesis has been shown. On the other hand, saprophytic staphylococci are able to displace pathogenic microflora in atopic dermatitis (Nakatsuji et al., 2017). However, there are only few investigations for many combinations of bacteria, such as E. coli, Klebsiella, Bacilli etc. As well, less data on inter-bacterial interactions in such consortia are known. Understanding the mechanisms of interspecies interactions in mixed consortia of pathogenic bacteria will allow adjusting the recommendations for the use of different antibiotics depending on the microbial composition of the infection and improve the efficacy of the therapy.

\section{Materials and methods}

Staphylococcus aureus (ATCC 29213), Pseudomonas aeruginosa (ATCC 27853), Escherichia coli (MG1655), Klebsiella pneumonia (clinical isolate) were used in this study. For the biofilm assay bacteria were grown under static conditions at $35^{\circ} \mathrm{C}$ for 48 hours in BM broth (Kayumov et al., 2015a; 2015b; Baidamshina et al., 2017). The mannitol-salt agar, Endo agar and Cetrimide agar were used to distinguish bacteria in mixed cultures. Biofilm formation was assessed in 24-well polystirol plates (Eppendorf) by staining with crystal violet (Sharafutdinov et al., 2017). The minimum inhibitory concentration (MIC) of antimicrobials was determined by the broth microdilution method according to the recommendation of EUCAST. The viability of cells was assessed by CFUs count (Sharafutdinov et al., 2017). Experiments were carried out in three biological repeats with three technical repeats in each of them.

\section{Results and discussion}

We have shown that $S$. aureus and $P$. aeruginosa form a stable mixed biofilm in vitro with prevalent location of $S$. aureus in upper layers of the biofilm.

*Corresponding author.

E-mail address: kairatr@yandex.ru (A. R. Kayumov)

(C) Author(s) 2020. This work is distributed under the Creative Commons Attribution 4.0 License. 
When mixed biofilms were treated with $S$. aureusspecific antimicrobials like vancomycin, tetracycline, ampicillin, and ceftriaxone, $P$. aeruginosa cells in the biofilm remained viable regardless of the presence of antibacterial substances. At the same time, $S$. aureus cells also survived, and were located in bottom layers of the biofilm suggesting that $S$. aureus is able to integrate into the matrix of $P$. aerugunosa biofilm and form separate microcolonies within it, and thus avoid of antimirobial drugs. On the other hand, antibiotics such as ciprofloxacin and aminoglycosides, which are effective against both strains, led to no re-arrangements of bacterial distribution in mixed biofilm and 10-fold stronger effect in compare with biofilms of monocultures. Similar results were obtained in experiments on mixed cultures of $S$. aureus and $K$. pneumoniae, $S$. aureus and $E$. coli. These data confirm that interactions between bacteria in the mixed biofilm significantly change the sensitivity to antibiotics of the microbial consortium. Moreover, these changes are bi-directional and the correct choice of antimicrobials determines whether the interbacterial interaction pattern will diminish or enhance the efficacy of antimicrobial.

\section{Acknowledgments}

This work was supported by the Russian Foundation for Basic Research, grant No 20-04-00247 and Russian Government Program of Competitive
Development of Kazan Federal University. This work was supported by RFBR grants (No 19-34-50042 mol nr, No 17-00-00456, No 19-34-90061).

\section{References}

Baidamshina D.R., Trizna E.Y., Holyavka M.G. et al. 2017. Targeting microbial biofilms using Ficin, a nonspecific plant protease. Scientific Reports 7. DOI: 10.1038/srep46068

Kayumov A.R., Khakimullina E.N., Sharafutdinov I.S. et al. 2015a. Inhibition of biofilm formation in Bacillus subtilis by new halogenated furanones. Journal of Antibiotics 68: 297-301. DOI: 10.1038/ja.2014.143

Kayumov A.R., Nureeva A.A., Trizna E.Y. et al. 2015b. New derivatives of pyridoxine exhibit high antibacterial activity against biofilm-embedded Staphylococcus cells. Biomed Research International 2015. DOI: 10.1155/2015/890968

Nakatsuji T., Chen T.H., Narala S. et al. 2017. Antimicrobials from human skin commensal bacteria protect against. Science Translational Medicine 9. DOI: 10.1126/ scitranslmed.aah4680

Sanchez-Vizuete P., Orgaz B., Aymerich S. et al. 2015. Pathogens protection against the action of disinfectants in multispecies biofilms. Frontiers in Microbiology 6. DOI: 10.3389/fmicb.2015.00705

Sharafutdinov I.S., Trizna E.Y., Baidamshina D.R. et al. 2017. Antimicrobial effects of sulfonyl derivative of 25H-Furanone against planktonic and biofilm associated Methicillin-resistant and -susceptible Staphylococcus aureus. Frontiers in Microbiology 8. DOI: 10.3389/fmicb.2017.02246 